\title{
Tide-induced magnetic signals and their errors derived from CHAMP and Swarm satellite magnetometer observations
}

Jan Saynisch-Wagner ${ }^{1 *} \mathbb{D}$, Julien Baerenzung ${ }^{2}$, Aaron Hornschild ${ }^{1}$, Christopher Irrgang $^{1}$ and Maik Thomas ${ }^{1,3}$

\begin{abstract}
Satellite-measured tidal magnetic signals are of growing importance. These fields are mainly used to infer Earth's mantle conductivity, but also to derive changes in the oceanic heat content. We present a new Kalman filter-based method to derive tidal magnetic fields from satellite magnetometers: KALMAG. The method's advantage is that it allows to study a precisely estimated posterior error covariance matrix. We present the results of a simultaneous estimation of the magnetic signals of 8 major tides from 17 years of Swarm and CHAMP data. For the first time, robustly derived posterior error distributions are reported along with the reported tidal magnetic fields. The results are compared to other estimates that are either based on numerical forward models or on satellite inversions of the same data. For all comparisons, maximal differences and the corresponding globally averaged RMSE are reported. We found that the inter-product differences are comparable with the KALMAG-based errors only in a global mean sense. Here, all approaches give values of the same order, e.g., $0.09 \mathrm{nT}-0.14 \mathrm{nT}$ for M2. Locally, the KALMAG posterior errors are up to one order smaller than the inter-product differences, e.g., $0.12 \mathrm{nT}$ vs. $0.96 \mathrm{nT}$ for M2.
\end{abstract}

Keywords: Tides, Electromagnetic induction, Error covariance, Satellite magnetometer observations Graphical Abstract forward model KALMAG estimate

M2
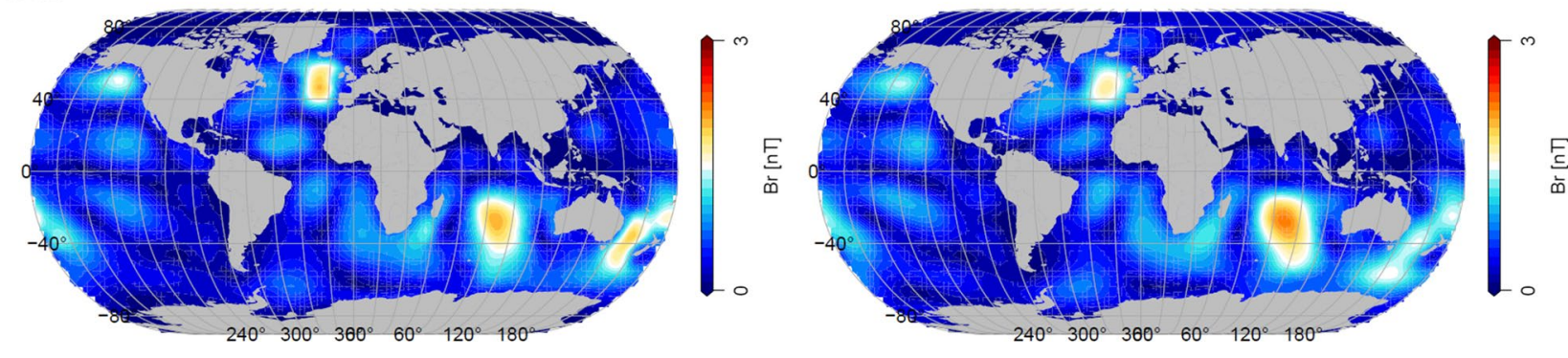

*Correspondence: saynisch@gfz-potsdam.de

1 Earth System Modelling, Helmholtz Centre Potsdam, GFZ German Research Centre, Potsdam, Germany

Full list of author information is available at the end of the article

\section{Introduction}

Electromagnetic (EM) tidal signals (EMTS) originate in the periodic movement of a conducting medium with respect to Earth's ambient magnetic field, e.g., the oceanic or the ionospheric dynamo (Larsen 1968; Malin 1970; 
Schnepf et al. 2014). Over long time, EMTS were available only point-wise, e.g., from terrestrial magnetometer stations (Kuvshinov et al. 2006; Maus and Kuvshinov 2004). In the beginning of the century, it became possible to detect global EMTS fields of the M2 tide by the use of the CHAMP satellite magnetometers (Tyler et al. 2003; Sabaka et al. 2015). With the still ongoing Swarm satellite magnetometer mission, EMTS of more tidal constituents (N2, O1) are observable (Sabaka et al. 2020; Grayver and Olsen 2019). Satellite-detected EMTS fields become increasingly important where electrical conductivity related information about the Earth's upper mantle (Grayver et al. 2016, 2017; Guzavina et al. 2019; Kuvshinov et al. 2021) or the world oceans (Saynisch et al. 2017; Irrgang et al. 2019) are to be inferred. Furthermore, EMTS can worsen the recovery of other contributions to Earth's observed magnetic signals (Guzavina et al. 2018).

Apart from the EMTS themselves, not much is published about the errors of these observation-based EMTS estimates. Surely, the quality of EMTS-derived quantities as mantle conductivity rely strongly on the accuracy of the EMTS observations. Especially for the assessment of EMTS temporal variations (Petereit et al. 2019; Irrgang et al. 2019; Saynisch-Wagner et al. 2020), reliable values of EMTS errors are missing.

The quality of the EM observation-based EMTS estimates is usually assessed by their similarity to modeled EMTS (e.g., Kuvshinov et al. 2006; Schnepf et al. 2014). For example, modeled oceanic EMTS use oceanographic products like the World Ocean Atlas (Zweng et al. 2018; Locarnini et al. 2018; Tyler et al. 2017) in combination with models of tidal transports (e.g., Egbert and Erofeeva 2002) and models of Earth's core field (e.g., Alken et al. 2021) to derive tidally generated electric currents. The corresponding Maxwell equations are then numerically solved in Earth's conducting environment (Püthe et al. 2015; Kuvshinov 2008). However, from differences between modeled and observed EMTS alone, no usable error estimates for the latter are derivable. The main reason is that the modeled EMTS include many error sources (e.g., Schnepf et al. 2014) that originate from uncertainties in the forcing data (e.g., Kuvshinov et al. 2006; Saynisch et al. 2018; Irrgang et al. 2019) and uncertainties in solving the EM induction problem (Sachl et al. 2019; Velimsky et al. 2018). Another reason is that, EM observations do observe a larger dynamical system than the one tidal forward models describe (Schnepf et al. 2018; Velímsky et al. 2021).

Other studies (Sabaka et al. 2016; Grayver and Olsen 2019; Sabaka et al. 2020) compare their observation-based EMTS estimates to previously published observation-based EMTS estimates that differ by observation time or/and observation system. However, these comparisons remain on a more or less qualitative level and report correlations rather than root mean squared errors (RMSE). In this paper, we want to add to this ongoing discussion by (a) adding an additional inversion method (a Kalman filter) to the available products and by (b) reporting the method's posterior-error estimates. In addition, the KALMAG-based EMTS estimates are compared to previously published observation-based inversions as well as to forward model-based EMTS estimates. For all comparisons, maximal differences and the corresponding globally averaged RMSE are reported. The study should help shift the focus of attention also to the errors of the satellite magnetometer observations (and derived products) and motivate the publication of error estimates from other groups and approaches.

\section{Data}

Swarm and CHAMP satellite magnetometer data are used to infer EMTS from the Earth's magnetic field measurements. The measurements cover a time window of mid-2000 to the end of 2020, with a 3-year gap between the two satellite missions (from Sept. 2010 to Nov. 2013). The sample interval is $10 \mathrm{~s}$. The data selection criteria are described in detail in Baerenzung et al. (2020), i.e., times with a $K_{p}$ index above $2^{0}$ are omitted, between magnetic latitudes of $60 \mathrm{~N}$ and $60 \mathrm{~S}$ only nighttime data (when the sun is below the horizon) are used and the measurements from Swarm-C are omitted entirely.

\section{Methods}

EM measurements are superpositions of several sources of electromagnetic signals, such as the dynamo of Earth's core and the processes in the ionosphere. To separate the EMTS from other non-tidal magnetic sources, the KALMAG framework is used (Baerenzung et al. 2020). The KALMAG framework describes and parameterizes each individual EM source by statistical (spatial and temporal) properties through a prior-covariance matrix. Its spatial part derives from an imposed (or estimated) spherical harmonic (SH) spectrum under the assumption of isotropy. The different sources are composed by the Earth's core, the lithosphere, the ionosphere, the magnetosphere, but also field-aligned currents. For a full list of EM sources used in KALMAG, see Tab. 1 of Baerenzung et al. (2020). The temporal evolution of each source is parameterized and modeled by an associated autoregressive process (Gillet et al. 2013; Bärenzung et al. 2018). Each individual EM source is sequentially propagated through time and every 2 min all sources are superimposed and the satellite observations are assimilated by a Kalman filter (Kalman 1960). As a result, the prior information is updated by the satellite observations and over time all 
EM sources become fitted to the observations. In a final Kalman-smoother step, the fitted sources can be propagated backwards in time to cover the whole assimilation period. The EM sources for core and lithospheric field and their temporal progression (from year 1900 to 2026) as they are estimated by the KALMAG approach are available online (https://ionocovar.agnld.uni-potsd am.de/Kalmag/).

For this study, the KALMAG framework was extended to incorporate harmonic tidal signals for 8 major tides (see Table 1). Within our approach, the tides are described entirely by their frequency and approximate first guess $\mathrm{SH}$ power spectra. In contrast to the other EM sources within KALMAG, the EMTS are not modeled as autoregressive processes. The physical properties of the ocean are assumed to be static over the entire assimilation period, and the different EMTS are only temporally constrained by the period of their harmonic oscillations. The KALMAG model was configured to incorporate the EMTS of Table 1 up to $\mathrm{SH}$ degree and order of 30 . This value is more than enough to cover the dominant EMTS features (see SH spectra in Fig. 5).

For modeled fields of the individual EMTS, the 1D mantle conductivity of Grayver et al. (2017), sediments from Laske and Masters (1997) and Everett et al. (2003), sea water conductivities calculated from the World Ocean Atlas (WOA18, Zweng et al. 2018; Locarnini et al. 2018) are used in combination with tidal transports that are based on satellite altimetry (TPXO8atlas, Egbert and Erofeeva 2002) and the IGRF-13 Earth magnetic field (Alken et al. 2021) to generate tidal electric current densities (cf., Saynisch et al. 2018; SaynischWagner et al. 2020). All fields except TPXO8-atlas are available on a $1 \times 1$ degree grid. We kept that resolution since it is sufficient to avoid resolution-based biases in the solutions of the induction equations (cf., Sachl et al. 2019). For this, TPXO8-atlas had to be interpolated down from its native $1 / 30$ degree resolution to the

Table 1 Tidal constituents used within the KALMAG approach

\begin{tabular}{lll}
\hline Name & Origin & Period [h] \\
\hline M2 & Principal lunar semidiurnal & 12.42060122 \\
S2 & Principal solar diurnal & 12.0 \\
K1 & Lunar diurnal & 23.93446961 \\
N2 & Larger lunar elliptic semidiurnal & 12.65834751 \\
O1 & Lunar diurnal & 25.81933871 \\
K2 & Lunisolar semidiurnal & 11.9672348 \\
P1 & Solar diurnal & 24.06589023 \\
Q1 & Larger lunar elliptic diurnal & 26.8683566 \\
\hline
\end{tabular}

$1 x 1$ degree resolution of this study. Subsequently, the respective Maxwell equations are solved by the X3DG solver of Kuvshinov (2008) to calculate the respective EMTS.

\section{Results and discussion}

The KALMAG estimates of the radial magnetic field of the tides listed in Table 1 are compared to the modeled EMTS in Figs. 1 and 2. The left panel of Figs. 1 and 2 shows the model-based fields and the right panel shows the respective KALMAG mean predictions. For the latter, the tides with periods close to $12 \mathrm{~h}$ and $24 \mathrm{~h}$ (S2, K1, P1) are dominated by the zonal bands typical of atmospheric-ionospheric processes, i.e., processes not confined by continental bounds (cf. also Schnepf et al. 2018). These large zonal band-like structures are detected from KALMAG, but are absent in the forward models. The reason is that the solar tides as $\mathrm{S} 2$ and $\mathrm{P} 1$ have a far larger impact on the atmospheric induction than on the oceanic induction. As explained in the previous section, the modeled fields are calculated from WOA18, TPXO8-atlas and IGRF-13. While the KALMAG approach detects all cumulative EM-signals on the prescribed tidal frequencies whether they originate in the oceans or not, the forward model fields consider only the tidal induction by the oceans. Consequently, discrepancies caused by physical reasons between both estimates have to be expected. Nonetheless, the oceanic contribution is also visible in S2, e.g., in the larger Indian Ocean area.

In case of the lunar tide K1, the reason for the discrepancies has to be different from the solar tides as S2. For $\mathrm{K} 1$, a separability problem is evident where harmonics of S2 and the P1 itself are not separable with the used data (see also, Love and Rigler 2014). Especially, considering that due to the daytime large data gaps occur with the same frequency. Please note, that since the amount of satellite data and quality varies over the globe, not only the EMTS errors (Fig. 4), but also the frequency separability varies over the globe as can be assessed by studying the KALMAG posterior error covariances among the EMTS (not plotted).

Where the tidal frequency is dominated by oceanic EM induction (M2, N2, O1 and Q1), the KALMAG estimates show the blob-like features typical for oceanic processes which have to resonate within their restricted basins. Some fields as K2 and the Q1 show a comparable spatial characteristic as the forward models, but the patterns are not always in the right locations. On the one hand, as already discussed, some of the reasons are physically based, e.g., for K2. At least in part, since with K2 a mix of both physical and separability reasons can be assumed (Love and Rigler 2014). On the other hand, some signals have to be considered as below the level of detectability 


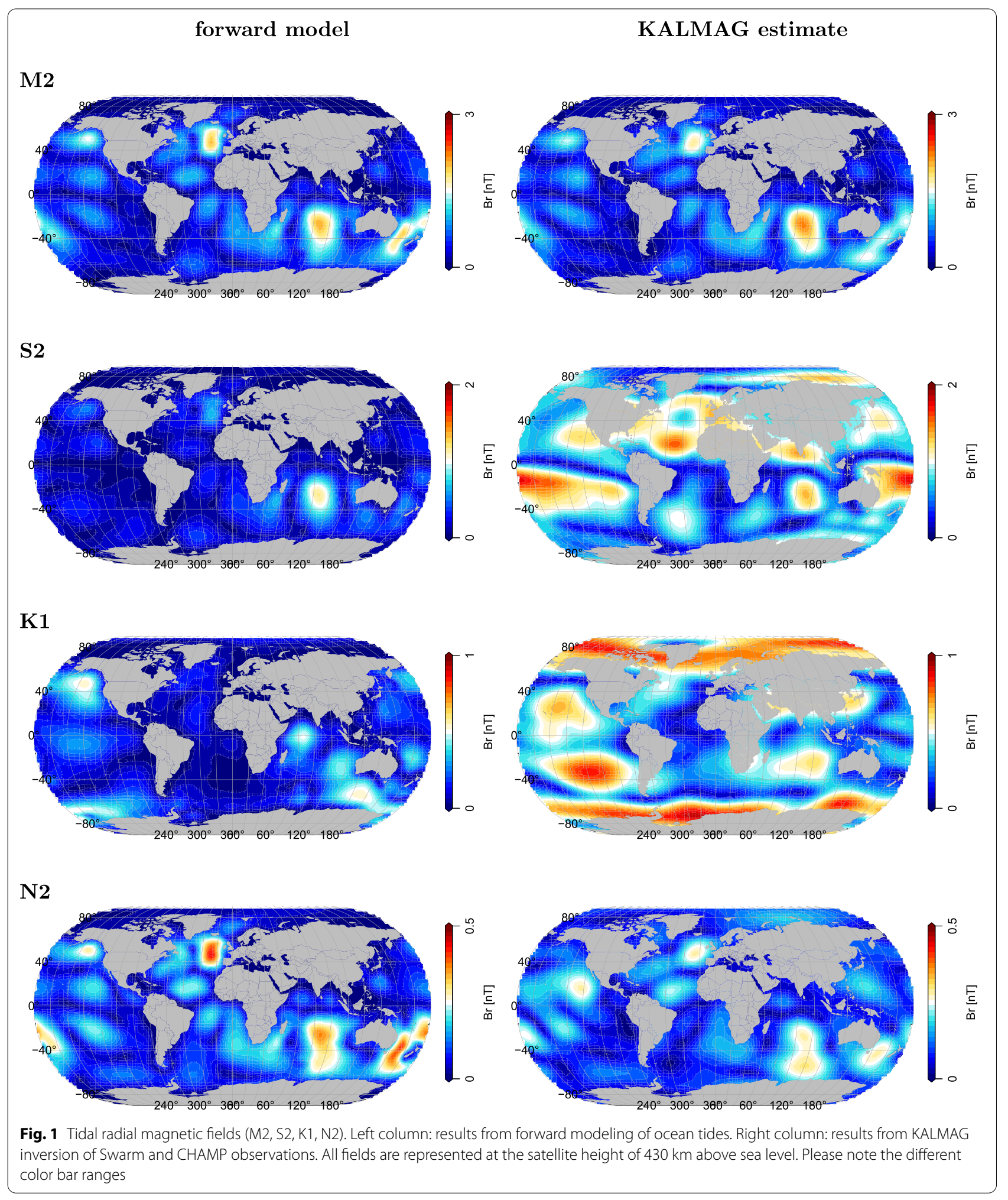




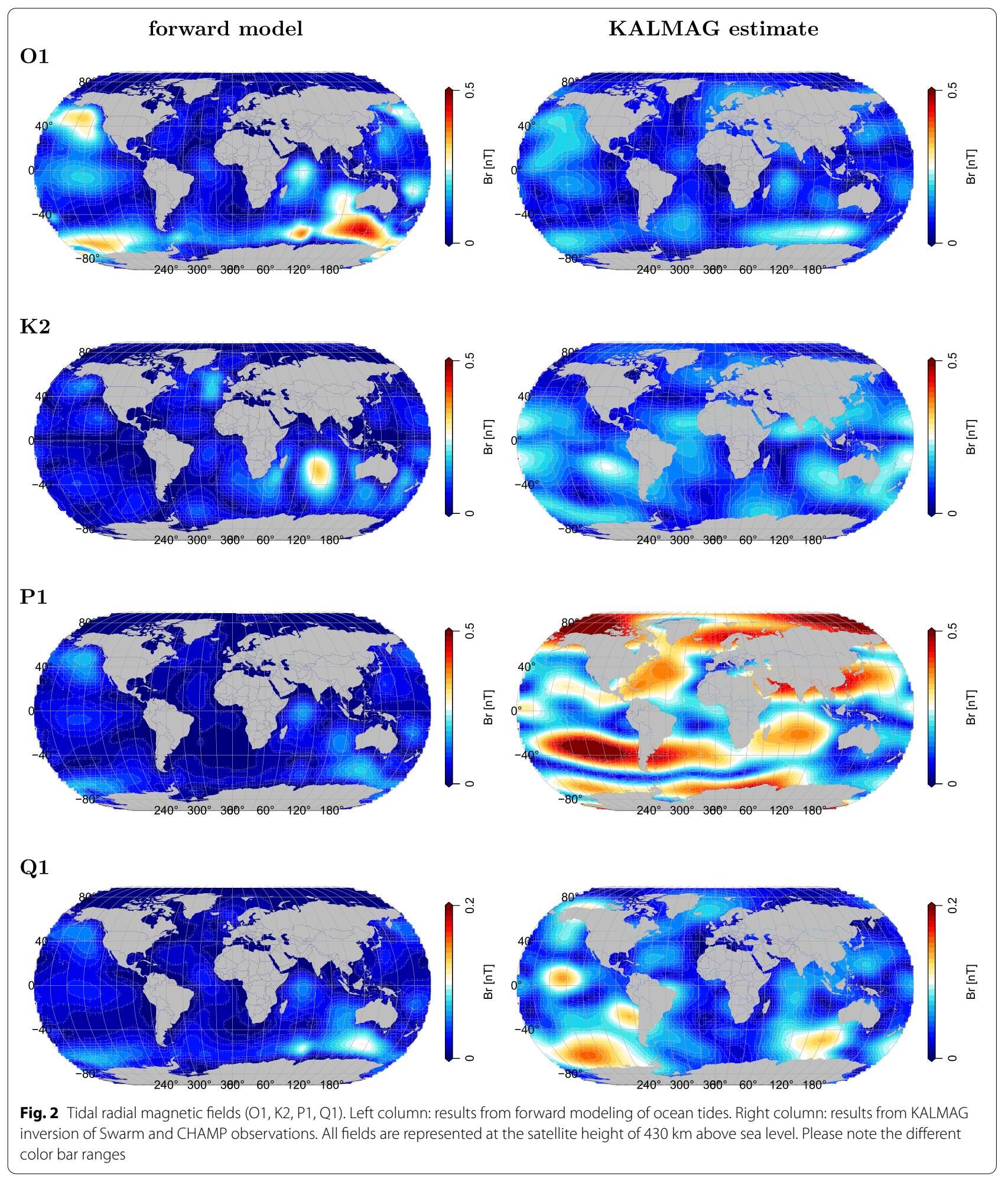


for the available data and the noise levels of the observations (Q1, P1, see also Fig. 4).

However, there are differences between forward models and KALMAG estimates which cannot be easily attributed to the mentioned physical reasons, separability reasons or to the observation's noise level. Even for wellfitting fields such as M2 and N2, KALMAG shows different amplitudes compared to the forward models. For M2, the main induction areas around New Zealand and the North Atlantic are up to $0.63 \mathrm{nT}$ smaller in KALMAG than in the forward model while the Indian Ocean shows a slightly $(0.54 \mathrm{nT})$ higher signal in the KALMAG estimate. The respective globally averaged RMSE of the M2 fields amount to $0.09 \mathrm{nT}$ at satellite height. In the case of N2, the KALMAG estimate is overall smaller. Locally, the discrepancies reach up to $0.18 \mathrm{nT}$ (RMSE: $0.05 \mathrm{nT}$ ). These different values probably have to be explained by the errors of the forward models. Saynisch et al. (2018) report that assimilative tidal models as the here used TPXO8-atlas can locally lead to errors of $0.1-0.3 \mathrm{nT}$ for the radial magnetic component of the M2 EMTS. These values are considerably smaller than the differences reported here. However, the published EMTS errors arise only due to the errors in the tidal model's transports. Additional errors from atmospheric (co-)excitations (Schnepf et al. 2018) and errors in the Earth's core field including its secular variation as well as in the conductivity distribution of sediments, mantle, crust and oceans are not considered in Saynisch et al. (2018). These additional errors are in part responsible for the high differences reported here between KALMAG and the forward models. Further sources of uncertainty are introduced in the latter by approximations in the used solver for the Maxwell equations including different resolutions of the underlying tidal transports (Sachl et al. 2019; Velimsky et al. 2018). Consequently, the forward model fields cannot be used as an ultimate benchmark tool to quantify the quality of the KALMAG estimates.
Consequently to further evaluate the KALMAG results, we compare them with other satellite-based EMTS estimates (see Fig. 3). The Comprehensive Model 5 (CM5) of Sabaka et al. (2015) inverts 12 years of CHAMP data together with Orsted, SAC-C and observatory data for various EM sources from core, lithosphere, ionosphere, magnetosphere and also the EMTS of the M2 ocean tide. Likewise, the Comprehensive Inversion Year 4 (CIY4) of Sabaka et al. (2018) inverts the first 4 years of Swarm observations for the same sources. By comparing the CIY4/CM5 M2 results in Fig. 3 with the top row of Fig. 1, i.e., the forward model/ KALMAG M2 results, one can see that all four fields share the overall global pattern and general magnitude of the M2 EMTS. Differences between KALMAG and CIY4 amount up to $0.77 \mathrm{nT}$ (RMSE: $0.13 \mathrm{nT}$ ) and are mostly located in the Southern Ocean below the Indian Ocean and in the North Atlantic. The respective differences between KALMAG and CM5 can amount up to $0.96 \mathrm{nT}$ (RMSE: 0.14 nT) and are mostly located in the Arctic Ocean and around New Zealand.

Compared to the forward model estimates, all fields that are based on satellite magnetometer observations show slightly different amplitudes. At satellite height, the M2 estimates of CIY4 shows differences of up to $0.75 \mathrm{nT}$ (RMSE: $0.14 \mathrm{nT}$ ) while CM5 shows differences of up to $0.84 \mathrm{nT}$ (RMSE: $0.13 \mathrm{nT}$ ) compared to the forward model. Consequently, at least for M2 the KALMAG results fit slightly better to the forward model than the CM5 and the CIY4 estimates. However, considering the previously discussed uncertainties included in the forward models, it is clear that such a comparison cannot be used to benchmark the products among each other. Furthermore, the forward model used to calculate these differences is not recalculated to match the specific CM5 or CIY4 time period. A forward model exactly calculated for the CM5/CIY4 time periods might show slightly different discrepancies compared with the CM5/CYI4 estimates

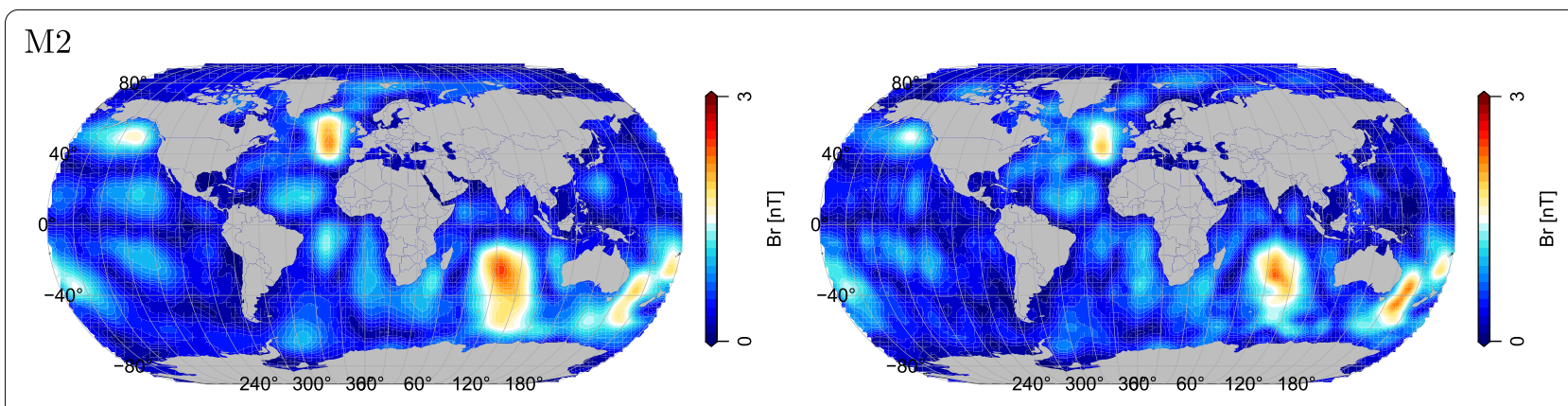

Fig. 3 Tidal radial magnetic field (M2). Left column: results from CIY4 (Sabaka et al. 2018), i.e., 4 years of Swarm data. Right column: results from CM5 (Sabaka et al. 2015), i.e., mostly 12 years of CHAMP data. All fields are represented at the satellite height of $430 \mathrm{~km}$ above sea level 
Table 2 Posterior errors from the KALMAG Kalman filter approach for the tidal constituents of Table 1

\begin{tabular}{lll}
\hline Name & Global mean error [nT] & $\begin{array}{l}\text { Local } \\
\text { max. error } \\
{[\mathbf{n T}]}\end{array}$ \\
\hline M2 & 0.09 & 0.12 \\
S2 & 0.09 & 0.11 \\
K1 & 0.08 & 0.09 \\
N2 & 0.06 & 0.08 \\
O1 & 0.05 & 0.07 \\
K2 & 0.05 & 0.07 \\
P1 & 0.06 & 0.07 \\
Q1 & 0.04 & 0.06 \\
\hline
\end{tabular}

(cf., Sabaka et al. 2015, 2018; Grayver and Olsen 2019; Sabaka et al. 2020).

Table 2 shows the EMTS posterior errors as they are estimated by the KALMAG Kalman filter approach. Since KALMAG fits its EM sources in SH space, the respective error-covariance matrix describes the $\mathrm{SH}$ space as well. To transfer the posterior error information onto geographical coordinates, the posterior errorcovariance matrix was sampled (a thousand times) in $\mathrm{SH}$ space and each field was transferred separately to geographical coordinates. Subsequently, the standard deviation of these gridded fields was calculated. Compared to the discussed differences between KALMAG, CM5/CIY4 and the forward models, the posterior errors are locally much smaller. For example, the M2 resp. N2 differences between KALMAG and forward model can locally reach $0.63 \mathrm{nT}$ resp. $0.18 \mathrm{nT}$ while the KALMAG posterior errors only reach $0.12 \mathrm{nT}$ resp. $0.08 \mathrm{nT}$. Again, discrepancies at this point are not worrisome since in contrast to KALMAG, the used forward models do not include atmospheric contributions. As a side note, KALMAG in the used setup is not made to distinguish oceanic and atmospheric EMTS if they share the same frequency. In principle, this could be done within KALMAG as the sources are further specified and modeled by their radial distance to the Earth's center. Future studies might aim, to separate oceanic and ionospheric tides by their height of origin. However, in the current KALMAG setup there is not enough information in the used observations to separate two sources by their height of origin unless one is an internal field while the other is an external field, i.e., their respective heights of origin are separated by the satellite height.

We summarize that the estimates are comparable at least in the global average. For M2 resp. N2, the globally averaged RMSE between KALMAG and the forward models are $0.09 \mathrm{nT}$ resp. $0.05 \mathrm{nT}$ and thus comparable to
KALMAG's globally averaged posterior errors of $0.09 \mathrm{nT}$ resp. $0.06 \mathrm{nT}$.

Comparing the M2 posterior errors with the KALMAG differences to the other satellite-based estimates gives slightly different results. Remember, that the M2-EMTS from KALMAG and CM5 resp. CIY4 differ by $0.96 \mathrm{nT}$ resp. $0.77 \mathrm{nT}$ locally and by $0.14 \mathrm{nT}$ resp. $0.13 \mathrm{nT}$ in the global average. Consequently, both the local as well as the globally averaged differences are bigger than the KALMAG posterior error estimates. The reason for this is not entirely clear. CM5/CIY4 aim to fit only the M2 tide while KALMAG uses 8 tidal frequencies. It is clear that the number of simultaneously fitted tides affects the results (Schnepf et al. 2014, 2018; Love and Rigler 2014). The more tidal constituents are fitted, less signal from unfitted constituents can leak into the fitted frequencies. In addition, the satellite product differences and the reported standard deviations describe different quantities and are not directly comparable. The mentioned product differences describe only single realizations or draws of a random variable while the KALMAG posterior error standard deviation describes a statistical property of a random variable. It would be much more interesting to directly compare the so far unpublished posterior error distributions of the other products.

Some EMTS have the same posterior errors (e.g., M2 and $\mathrm{S} 2$ in Table 2) but related to their individual signal strength, the relative posterior errors differ. The spatial distribution of the KALMAG posterior errors is plotted in Fig. 4 with each color bar presenting 15\% of each tide's typical signal strength (cf., the ranges in Figs. 1, 2). One can clearly see that for the stronger EMTS, such as M2, $\mathrm{S} 2$ and K1, the posterior errors are relatively small. Obviously, the weaker the EMTS are, the worse the signal-tonoise ratio becomes. Furthermore, the posterior error patterns are very similar to the EMTS patterns themselves (compare Fig. 4 with Figs. 1, 2). In other words, for each tidal component, the errors grow with the local signal strength, an effect that is opposed to the previously mentioned signal-to-noise ratio.

Figure 5 depicts the spatial power spectra of the KALMAG results. Compared to the spectra from CM5 (M2) and Grayver and Olsen (2019) (M2, N2 and O1), the KALMAG estimates show overall comparable values and shape. One obvious main difference is the higher power of the CM5's M2 inversion in higher SH degrees and orders. Here, the CHAMP only inversion of CM5 must be considered erroneously (Sabaka et al. 2018). KALMAG and Grayver and Olsen (2019) agree very well for the M2 spectra. However, Grayver and Olsen (2019) show M2 signal strength up to SH degree and order of 28 while KALMAG signal power reach zero already around $\mathrm{SH}$ degree and order of 22 . The opposite must be stated 

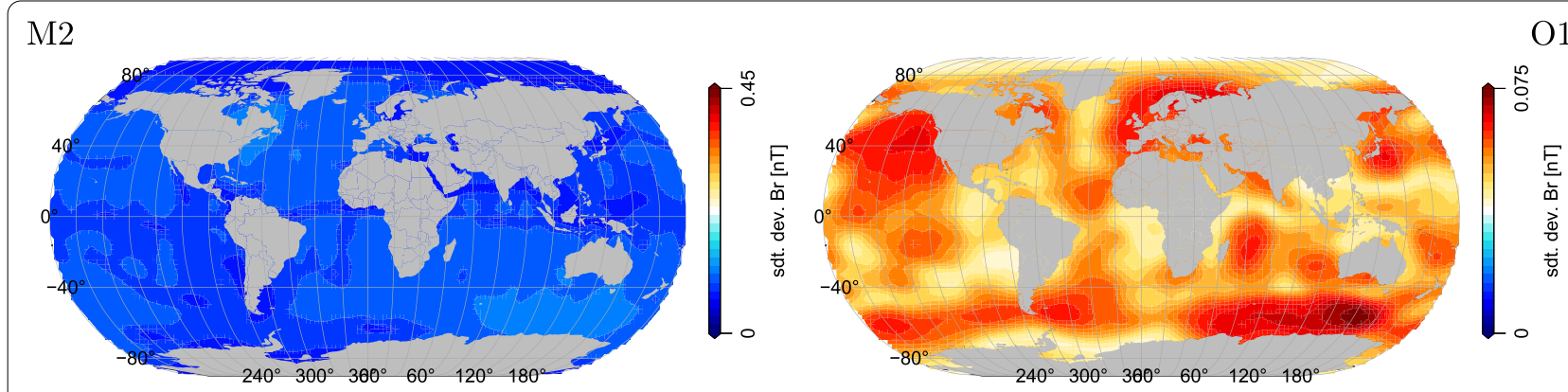

$\mathrm{S} 2$
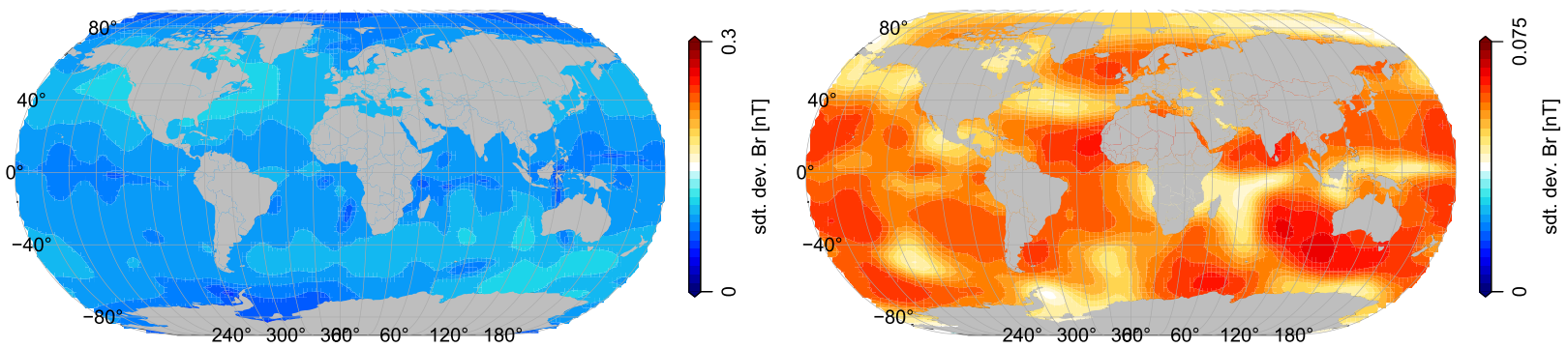

K1
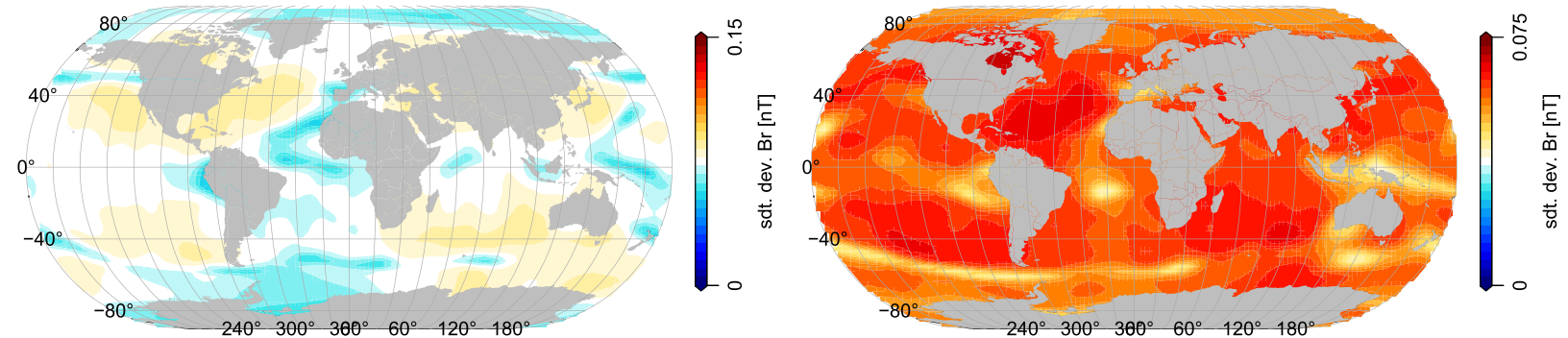

N2
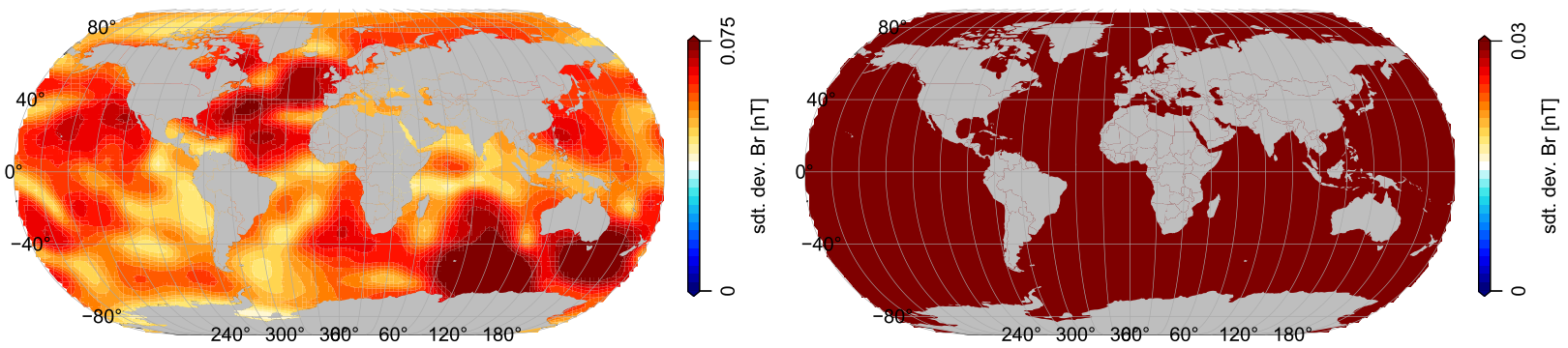

Fig. 4 Standard deviation of posterior errors of the tidal radial magnetic fields as estimated by the KALMAG Kalman filter. Left column: M2, S2, K1, N2. Right column: O1, K2, P1, Q1. All fields are represented at the satellite height of $430 \mathrm{~km}$ above sea level. Please note the different color bar ranges

for N2 and O1, where the KALMAG spectra show signal strength up to $\mathrm{SH}$ degree and order of 20 while the Grayver and Olsen (2019) estimates decay already at around 13. At least for KALMAG, we can state that these higher orders of N2 and O1 where Grayver and Olsen (2019) and KALMAG differ are in doubt since the KALMAG posterior errors are equally high as the KALMAG spectral power estimate (Fig. 5, thick dashed line). The same must be said about the SH degree and orders below 2 off KALMAG's N2 estimate and the respective differences to 

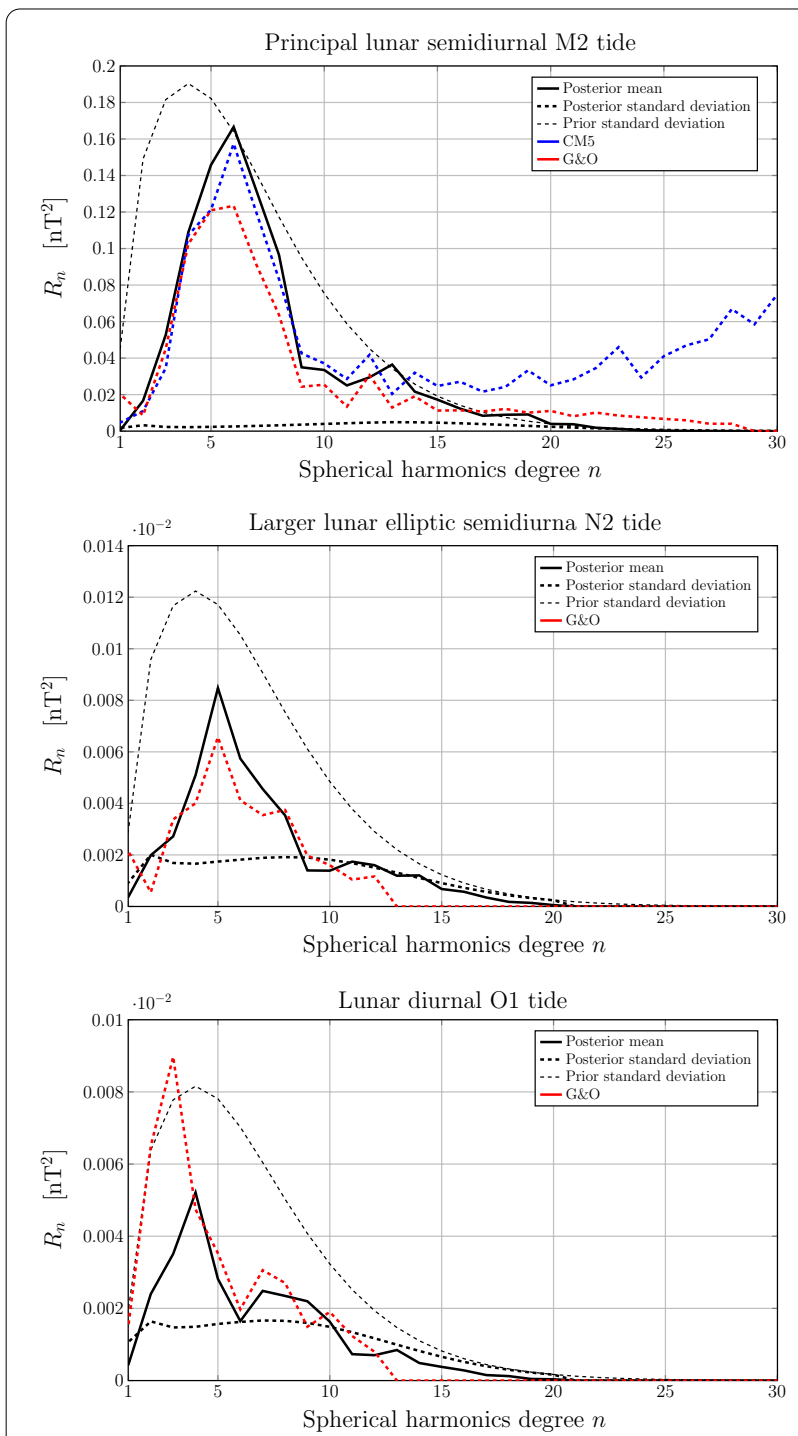

Fig. 5 Spatial SH power spectra of satellite-based EMTS estimates. From top to bottom: M2, N2 and O1. Black lines: KALMAG estimates (solid) with prior and posterior standard deviation (light and solid dashes). Blue: CM5 estimates from Sabaka et al. (2015), i.e., mostly 12 years of CHAMP data. Red: Estimates from Grayver and Olsen (2019), i.e., 15 years of CHAMP and Swarm data. For better comparability with the literature, all fields are represented at sea level (cf., Grayver and Olsen 2019; Sabaka et al. 2020)

Grayver and Olsen (2019). For O1, the KALMAG result shows a much weaker spectral power for $\mathrm{SH}$ degree and order of 4 and lower compared to the Grayver and Olsen (2019) result. Even considering KALMAG's relatively high error bars in that degree and order range, this difference must be considered significant. This difference is in part related to the already discussed spectral leakage. While Grayver and Olsen (2019) estimate 3 tides, the presented KALMAG approach estimates 8 tides simultaneously. Consequently, spectral leakage is less likely to occur in the latter case and the observed power is distributed among more tidal constituents. Please see also Sabaka et al. (2020) for an extensive spectral comparison between CM5, CM6, CIY4 and the Grayver and Olsen (2019) estimates. In addition, one can see how the spectra's assumed prior errors (light dashes) drop to an estimation of the posterior errors. For M2, the posterior errors are well below the signal strength up to $\mathrm{SH}$ degree and order of 20 (cf. Fig. 4). For N2 and O1, the posterior errors above (below) SH degree and order of around 10 (2) must already be considered as dominating.

\section{Conclusions}

The KALMAG posterior errors do not rely on specific differences to other products. On the one hand, the errors are more general than, e.g., calculating differences among the few existing satellite inversions. The posterior errors are also independent of the various assumptions made in the forward models as model resolution, conductivity distribution, tidal model, EM solver and more (Schnepf et al. 2014, 2018; Sachl et al. 2019; Saynisch et al. 2018; Velimsky et al. 2018). On the other hand, the errors are also specific in a sense that they are connected to the presented KALMAG approach and its output and they cannot be easily transferred to other approaches. Furthermore, the posterior errors do contain correlation information (not shown).

We conclude that the product-difference-based errors and the KALMAG posterior error estimates can and should complement each other. As a guidance, the posterior errors could be used as prior error estimates or as background errors for satellite-based inversion approaches or sensitivity studies that utilize EMTS anomalies. This is especially true for the global mean values as they are in the same order as the the global mean values of the discussed inter-product differences. The spatially resolved posterior errors can be downloaded (https://ionocovar.agnld.uni-potsdam.de/Kalmag/). The available products will be constantly updated.

\section{Summary}

Magnetic fields of eight major tides are extracted from the satellite magnetometer missions Swarm and CHAMP. The extraction is done using the Kalman filterbased software KALMAG that describes all involved EM sources entirely by their statistical properties. The approach detects electromagnetic contributions on the selected tidal frequencies using nighttime data. The signals of atmospheric or oceanic origin are not further distinguished.

We present the KALMAG approach's posterior error distributions and compare them to other products that 
are based on numerical models as well as on satellite inversions. We found that the inter-product differences are comparable with the KALMAG based errors only in a global mean sense. Here, both approaches give values of the same order, e.g., $0.09 \mathrm{nT}-0.14 \mathrm{nT}$, for M2. Locally, the KALMAG posterior errors can be one order smaller than the inter-product differences, e.g., $0.12 \mathrm{nT}$ vs. $0.96 \mathrm{nT}$, again for M2. Since the inter-product differences represent only a few realizations (or draws) of a random variable, the comparisons made to KALMAG's fully known posterior error covariance matrix can only be considered skewed. A comparison to posterior errors of other inversion products would be preferable.

To improve the presented approach, several strategies are envisioned. First, prior spatial information about the expected tidal pattern can be included in the KALMAG approach. Since this information possibly can only be derived from forward models, the prior information should be implemented in a way that it does not constrain the KALMAG inversion approach too much, e.g., it would be preferable to only use correlations of tidal spherical coefficients rather than covariances or even the exact values. Second, likewise generated cross-correlations among the individual tidal constituents could be incorporated to further constrain the KALMAG inversion.

\section{Acknowledgements \\ The authors thank for the opportunity to use data from the World Ocean Atlas (www.nodc.noaa.gov/OC5/woa18/), the TPXO tidal model (http://volkov. oce.orst.edu/tides/) and the IGRF-13 geomagnetic reference field. We thank Alexey Kuvshinov (kuvshinov@erdw.ethz.ch) for the opportunity to use the 3D EM induction solver X3DG and Terence Sabaka for providing the M2 tidal magnetic field products CM5 and CIY4.}

\section{Authors' contributions}

JS designed and conducted the study and wrote the majority of the paper. JB developed the KALMAG code and implemented the tidal sources. AH contributed by calculation of the forward model estimates. Cl prepare the comparison to the satellite-based CM5/CIY4 estimates. MT is involved with acquisition of funding and administration of the project. All authors did participate in reviewing of the paper. All authors read and approved the final manuscript.

\section{Funding}

Open Access funding enabled and organized by Projekt DEAL. This study was funded by the German Research Foundation (SPP1788, Dynamic Earth) and the Initiative and Networking Fund of the Helmholtz Association through the project "Advanced Earth System Modelling Capacity" (ESM).

\section{Availability of data and materials}

The described KALMAG-based EMTS and the respective posterior errors can be downloaded from https://ionocovar.agnld.uni-potsdam.de/Kalmag/. Swarm and CHAMP data can be downloaded from our institute at https:// www.gfz-potsdam.de/en/section/geomagnetism/infrastructure/. The Kp index can be downloaded at ftp://ftp.gfz-potsdam.de/pub/home/obs/kp-ap/. The KALMAG model presented here is available upon request.

\section{Competing interests}

The authors declare that they have no competing interests.

\section{Author details}

${ }^{1}$ Earth System Modelling, Helmholtz Centre Potsdam, GFZ German Research Centre, Potsdam, Germany. ${ }^{2}$ Potsdam University, Potsdam, Germany. ${ }^{3}$ Department of Earth Sciences, Institute of Meteorology, Freie Universität-Berlin, Berlin, Germany.

Received: 15 September 2021 Accepted: 8 December 2021 Published online: 20 December 2021

\section{References}

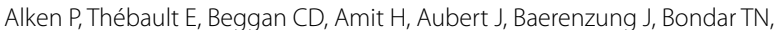
Brown WJ, Califf S, Chambodut A, Chulliat A, Cox GA, Finlay CC, Fournier A, Gillet N, Grayver A, Hammer MD, Holschneider M, Huder L, Hulot G, Jager T, Kloss C, Korte M, Kuang W, Kuvshinov A, Langlais B, Léger J-M, Lesur V, Livermore PW, Lowes FJ, Macmillan S, Magnes W, Mandea M, Marsal S, Matzka J, Metman MC, Minami T, Morschhauser A, Mound JE, Nair M, Nakano S, Olsen N, Pavón-Carrasco FJ, Petrov VG, Ropp G, Rother M, Sabaka TJ, Sanchez S, Saturnino D, Schnepf NR, Shen X, Stolle C, Tangborn A, Tøffner-Clausen L, Toh H, Torta JM, Varner J, Vervelidou F, Vigneron P, Wardinski I, Wicht J, Woods A, Yang Y, Zeren Z, Zhou B (2021) International geomagnetic reference field: the thirteenth generation. Earth, Planets and Space 73(1):49. https://doi.org/10.1186/s40623-020-01288-x

Baerenzung J, Holschneider M, Wicht J, Lesur V, Sanchez S (2020) The Kalmag model as a candidate for IGRF-13. Earth, Planets and Space 72(163):1-13. https://doi.org/10.1186/s40623-020-01295-y

Bärenzung J, Holschneider M, Wicht J, Sanchez S, Lesur V (2018) Modeling and predicting the short-term evolution of the geomagnetic field. J Geophys Res Solid Earth 123(6):4539-4560

Egbert GD, Erofeeva SY (2002) Efficient inverse modeling of barotropic ocean tides. J Atmos Ocean Tech. 19:183-204

Everett ME, Constable S, Constable CG (2003) Effects of near-surface conductance on global satellite induction responses. Geophys J Int. 153(1):277-286

Gillet N, Jault D, Finlay CC, Olsen N (2013) Stochastic modeling of the Earth's magnetic field: inversion for covariances over the observatory era. Geochem Geophys Geosyst. 14:766-786

Grayver AV, Olsen N (2019) The magnetic signatures of the M2, N2, and O1 oceanic tides observed in Swarm and CHAMP satellite magnetic data. Geophys Res Lett. 46(8):4230-4238

Grayver AV, Schnepf NR, Kuvshinov AV, Sabaka TJ, Manoj C, Olsen N (2016) Satellite tidal magnetic signals constrain oceanic lithosphere-asthenosphere boundary. Sci Adv 2(9):e1600798

Grayver AV, Munch FD, Kuvshinov AV, Khan A, Sabaka TJ, Tøffner-Clausen L (2017) Joint inversion of satellite-detected tidal and magnetospheric signals constrains electrical conductivity and water content of the upper mantle and transition zone. Geophys Res Lett. 44(12):6074-6081

Guzavina M, Grayver A, Kuvshinov A (2018) Do ocean tidal signals influence recovery of solar quiet variations? Earth, Planets and Space 70(1):5. https://doi.org/10.1186/s40623-017-0769-1

Guzavina M, Grayver A, Kuvshinov A (2019) Probing upper mantle electrical conductivity with daily magnetic variations using global-to-local transfer functions. Geophys J Int. 219(3):2125-2147

Irrgang C, Saynisch J, Thomas M (2019) Estimating global ocean heat content from tidal magnetic satellite observations. Sci Rep 9(7893):8

Kalman RE (1960) A new approach to linear filtering and prediction problems. Trans ASME J Basic Eng. 82(Series:D):35-45

Kuvshinov AV (2008) 3-D global induction in the oceans and solid Earth: recent progress in modeling magnetic and electric fields from sources of magnetospheric, ionospheric and oceanic origin. Surv Geophys 29(2):139-186

Kuvshinov A, Junge A, Utada H (2006) 3-D modelling the electric field due to ocean tidal flow and comparison with observations. Geophys Res Lett. https://doi.org/10.1029/2005GL025043

Kuvshinov A, Grayver A, Toffner-Clausen L, Olsen N (2021) Probing 3-D electrical conductivity of the mantle using 6 years of Swarm, CryoSat- 2 and observatory magnetic data and exploiting matrix Q-responses approach. Earth, Planets and Space 73(67):1880-5981. https://doi.org/10.1186/ s40623-020-01341-9

Larsen JC (1968) Electric and magnetic fields induced by deep sea tides. Geophys J R astr Soc. 16:47-70 
Laske G, Masters G (1997). A global digital map of sediment thickness. Eos Trans. AGU, 78(46), Fall Meet. Suppl

Locarnini RA, Mishonov AV, Baranova OK, Boyer TP, Zweng MM, Garcia HE, Reagan JR, Seidov D, Weathers K, Paver CR, Smolyar I (2018). World Ocean Atlas 2018, Volume 1: Temperature. Tech. rept. 81. NOAA

Love JJ, Rigler EJ (2014) The magnetic tides of Honolulu. Geophys J Int. 197(3):1335-1353

Malin SRC (1970) Separation of Lunar daily geomagnetic variations into parts of ionospheric and oceanic origin. Geophys J R Astr SOC. 21:447-455

Maus S, Kuvshinov A (2004) Ocean tidal signals in observatory and satellite magnetic measurements. Geophys Res Lett. https://doi.org/10.1029/ 2004GL020090

Petereit J, Saynisch J, Irrgang C, Thomas M (2019) Analysis of ocean tide induced magnetic fields derived from oceanic in-situ observations climate trends and the remarkable sensitivity of shelf regions. J Geophys Res Oceans 124(11):8257-8270

Püthe C, Kuvshinov A, Khan A, Olsen N (2015) A new model of Earth's radial conductivity structure derived from over $10 \mathrm{yr}$ of satellite and observatory magnetic data. Geophys J Int. 203(3):1864-1872

Sabaka TJ, Olsen N, Tyler RH, Kuvshinov A (2015) CM5, a pre-Swarm comprehensive geomagnetic field model derived from over $12 \mathrm{yr}$ of CHAMP, Orsted, SAC-C and observatory data. Geophys J Int. 200(3):1596-1626

Sabaka TJ, Tyler RH, Olsen N (2016) Extracting ocean-generated tidal magnetic signals from Swarm data through satellite gradiometry. Geophys Res Lett. 43(7):3237-3245

Sabaka TJ, Toffner-Clausen L, Olsen N, Finlay CC (2018) A comprehensive model of Earth's magnetic field determined from 4 years of Swarm satellite observations. Earth, Planets and Space. 70(130):26. https://doi.org/10. 1186/s40623-018-0896-3

Sabaka TJ, Tøffner-Clausen L, Olsen N, Finlay CC (2020) CM6: a comprehensive geomagnetic field model derived from both CHAMP and Swarm satellite observations. Earth, Planets and Space. https://doi.org/10.1186/s40623020-01210-5.pdf

Sachl L, Martinec Z, Velimsky J, Irrgang C, Petereit J, Saynisch J, Einspigel D, Schnepf NR (2019) Modelling of electromagnetic signatures of global ocean circulation: physical approximations and numerical issues. Earth, Planets and Space 71(1):58. https://doi.org/10.1186/s40623-019-1033-7

Saynisch J, Petereit J, Irrgang C, Thomas M (2017) Impact of oceanic warming on electromagnetic oceanic tidal signals: a CMIP5 climate model-based sensitivity study. Geophys Res Lett. 44(10):4994-5000

Saynisch J, Irrgang C, Thomas M (2018) Estimating ocean tide model uncertainties for electromagnetic inversion studies. Ann Geophys. 36(4):1009-1014

Saynisch-Wagner J, Petereit J, Irrgang C, Thomas M (2020) Phase changes of electromagnetic oceanic tidal signals. J Geophys Res Oceans 125(4):e2019JC015960

Schnepf NR, Manoj C, Kuvshinov A, Toh H, Maus S (2014) Tidal signals in oceanbottom magnetic measurements of the Northwestern Pacific: observation versus prediction. Geophys J Int. 198(2):1096-1110

Schnepf NR, Nair M, Maute A, Pedatella NM, Kuvshinov A, Richmond AD (2018) A comparison of model-based ionospheric and ocean tidal magnetic signals With observatory data. Geophys Res Lett. 45(15):7257-7267

Tyler RH, Maus S, Luhr H (2003) Satellite observations of magnetic fields due to ocean tidal flow. Science 299(5604):239-241

Tyler RH, Boyer TP, Minami T, Zweng MM, Reagan JR (2017) Electrical conductivity of the global ocean. Earth, Planets and Space 69(1):156. https://doi. org/10.1186/s40623-017-0739-7

Velimsky J, Grayver A, Kuvshinov A, Šachl L (2018) On the modelling of M2 tidal magnetic signatures: effects of physical approximations and numerical resolution. Earth Planets Space. https://doi.org/10.1186/s40623-018-0967-5

Velímsky J, Schnepf NR, Nair MC, Thomas NP (2021) Can seafloor voltage cables be used to study large-scale circulation? An investigation in the Pacific Ocean. Ocean Sci 17(1):383-392

Zweng MM, Reagan JR, Seidov D, Boyer TP, Locarnini RA, Garcia HE, Mishonov AV, Baranova OK, Weathers K, Paver CR, Smolyar I (2018). World Ocean Atlas 2018, Volume 2: Salinity. Tech. rept. 82. NOAA

\section{Publisher's Note}

Springer Nature remains neutral with regard to jurisdictional claims in published maps and institutional affiliations.

\section{Submit your manuscript to a SpringerOpen ${ }^{\circ}$ journal and benefit from:}

- Convenient online submission

- Rigorous peer review

- Open access: articles freely available online

- High visibility within the field

- Retaining the copyright to your article

Submit your next manuscript at $\boldsymbol{\nabla}$ springeropen.com 